\title{
Modeling and Automation of Water Purification Processes from Multicomponent Pollution
}

\author{
https://doi.org/10.31713/MCIT.2019.53
}

\author{
Andrii Safonyk \\ National University of Water and Environmental Engineering \\ Institute of Automatics, Cybernetics and Computer \\ Engineering \\ Rivne, Ukraine \\ a.p.safonyk@nuwm.edu.ua
}

\author{
Victoria Zhabchyk \\ National University of Water and Environmental Engineering \\ Institute of Automatics, Cybernetics and Computer \\ Engineering \\ Rivne, Ukraine Rivne, Ukraine \\ zhabchyk.v_ak16@nuwm.edu.ua
}

\author{
Olga Safonyk \\ Rivne Humanitarian Gymnasium \\ Rivne, Ukraine \\ olya.safonyk@gmail.com
}

\begin{abstract}
Mathematical models of the processes of cleaning liquids from multicomponent contamination by filtration, as well as diffusion-mass transfer perturbations and the development of numerical-asymptotic methods for solving the corresponding nonlinear regularly and singularly perturbed boundary value problems are shown. The construction of automation systems of corresponding treatment systems and complexes on the basis of solving model problems is presented.
\end{abstract}

Keywords - mathematical model, purification, filtration, impurities, magnetic water purification, biological water purification, water lighting.

\section{INTRODUCTION}

As a rule, when modeling fluid purification processes by filtering through porous [1] medium is being considered filters with uniform loading of a constant section in the mode of constant filtering speed. Besides filtering down the grain size with decreasing velocity along decreasing velocity - these are generally recognized methods of intensifying the work of filter facilities that are widely used in practice (separate attempts to consider uniformity of filter loading cannot be considered satisfactory because the extreme idealization of the relevant assumptions) [2]. Not installed structure of the coefficients appearing in mathematical models, as a function of the loading parameters, characteristics of the source fluid and its impurity particles, filter speeds, characteristics of the precipitate that is formed, etc.

The concentration of impurity particles at the inlet of the filter is almost always considered constant, although in real conditions, it usually changes. Impurities of filtered liquids are considered to be monodisperse and non-interacting, while they are almost always polydisperse and may interact under certain conditions. Account diffusions and mass transfer components don't take into, which, under certain conditions, may be significant. (For porous filter materials with a large pore conduction coefficient, in many cases, diffusion and mass transfer and reverse effects can be signification). There are no mathematical models that take into account the interaction of impurity particles during filtration. There are no mechanisms for creating automated control systems based on existing mathematical models.

\section{ANALYSIS OF PREVIOUS STUDIES}

In most cases [3-4], the tasks in question contained onecomponent process characteristics, but, as practice shows, contamination is multicomponent and depends on many factors, leading to entirely new, complicated tasks.

\section{STATEMENT OF THE PROBLEM}

Since most sewage treatment plants operate on manual control, this results in reduced productivity, increased cost of services, and "human factor". Automation of water treatment plants will allow to reduce labor costs for maintenance in increase of reliability of work of plants, to improve technical and economic indicators at the expense of optimization and intensification of technological processes, to provide water of guaranteed quality. Automatic filtering [5] of the filters results in a reduction of $10-25 \%$ present of specific and total costs of chemical reagents in water for their own use. Introduction of automation of management of water treatment plants allow 2-3 times to increase the productivity of these installations without capital costs for technological equipment in construction, which is the main factor that determines the feasibility of introducing of systems of automation of water treatment plants.

\section{THE MAIN MATERIAL OF THE ARTICLE}

Automated systems of magnetic (fig. 1-2), biological (fig. 3) water purification are developed in the work. 


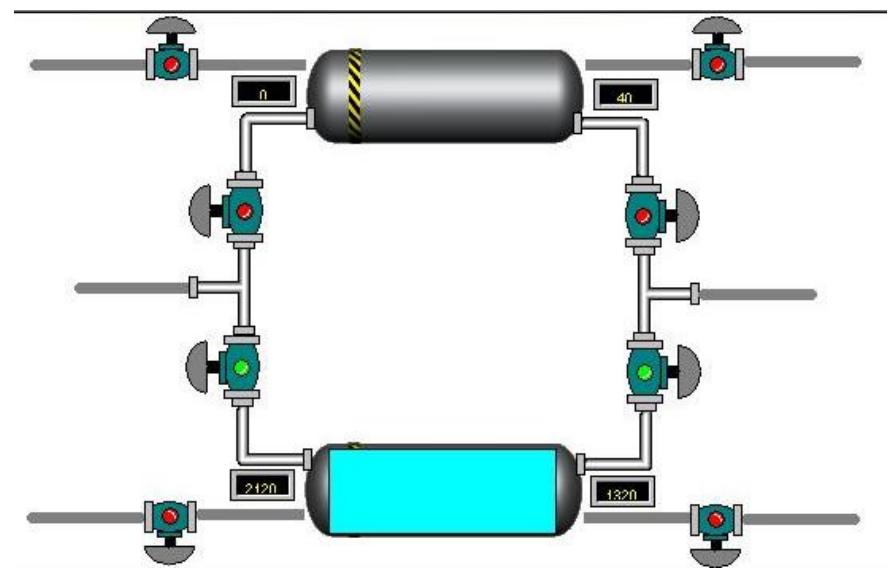

Figure 1. Mnemonic scheme of automation of the process of magnetic water purification of the boiler room

It is planned to build automated systems which allow on the basis of calculated dependencies, to curry out control of water treatment plants (fig. 4) and industrial treatment systems (fig. 5 ), which are currently operating in manual mode.

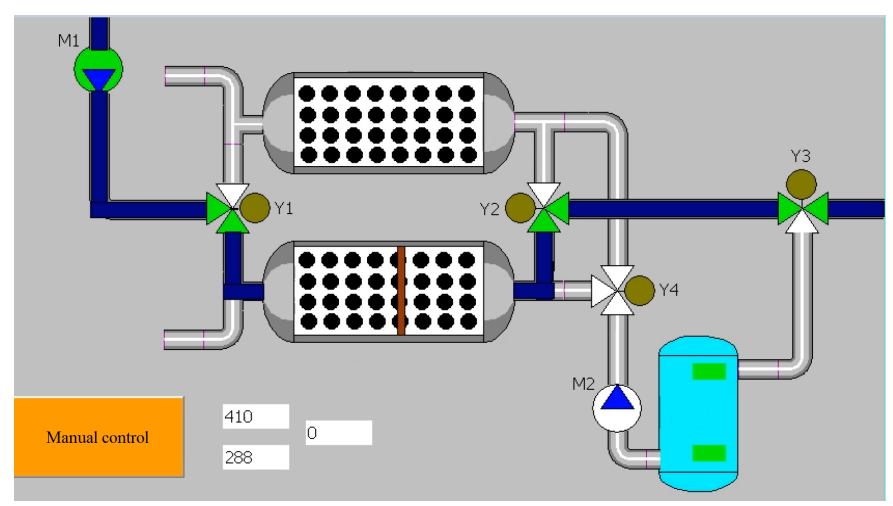

Figure 2. Scada system of magnetic cleaning of thermal power plants

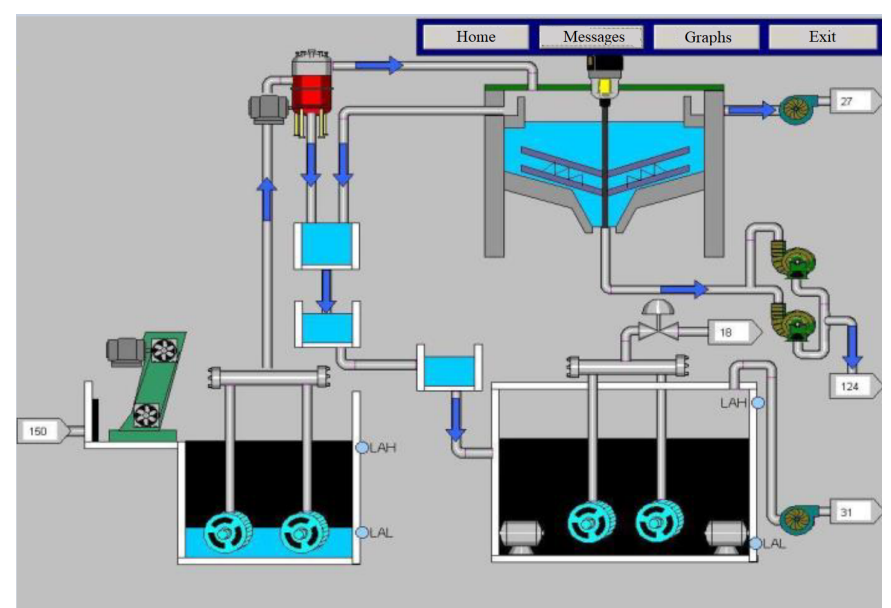

Figure 3. Scada biological water treatment system.

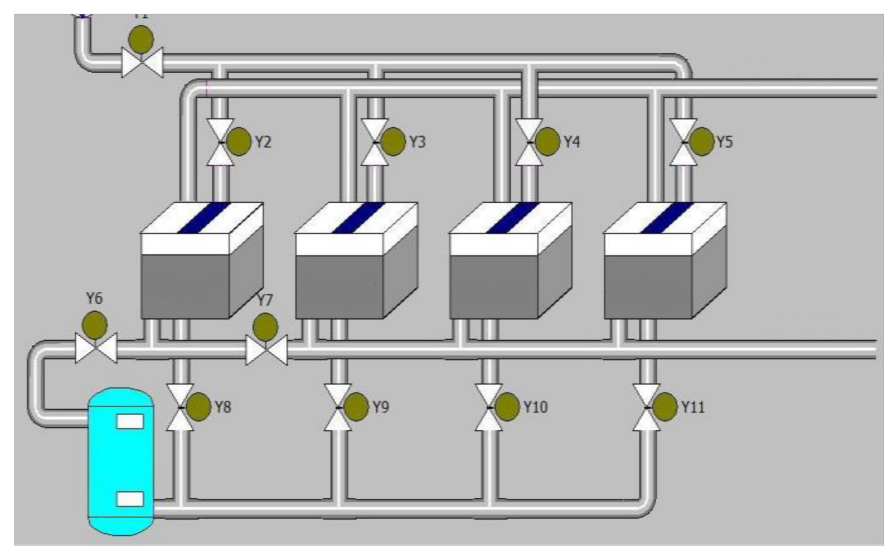

Figure 4. Automated water treatment plant control system

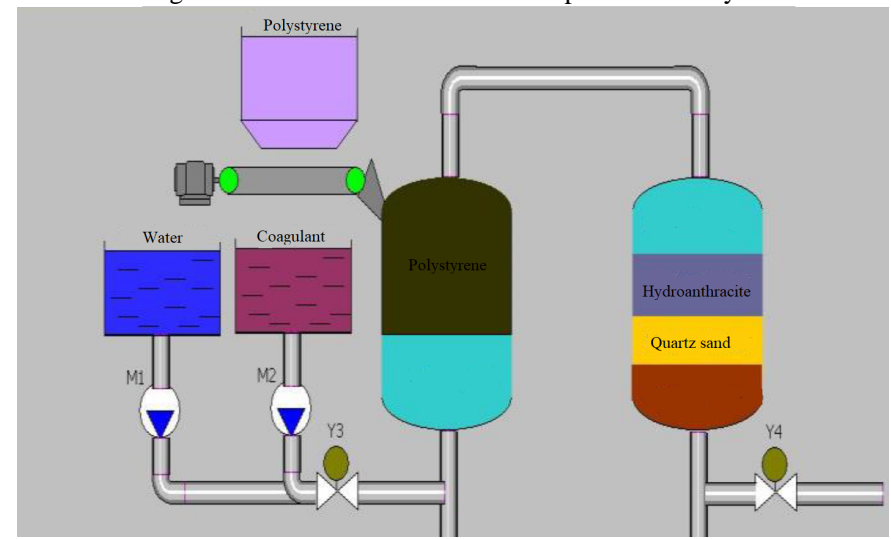

Figure 5. Automated water lighting system.

The processes of purification of liquids from multicomponent contamination by filtration in a porous medium [6] will be studied on the basis of a system of nonlinear differential equations:

$$
\begin{aligned}
& \frac{\partial}{\partial t}\left(\sigma(x, y, z, t, \rho) c_{1, l}\right)+\frac{\partial \rho_{i, l}}{\partial t}+\vec{v} \cdot \operatorname{grad}_{1, l}+f_{i, l}\left(x, y, z, t, c_{1, l}, \rho_{i, l}\right)= \\
& =\sum_{k=1}^{n}\left(\operatorname{div}\left(D_{k l}\left(x, y, z, t, c_{1, l}(x, y, z, t), c_{1, l}(x, y, z, t-\tau), b\right)\left(\operatorname{gradc}_{1, l}\right)\right)\right), \\
& \frac{\partial}{\partial t}\left(\sigma(x, y, z, t, \rho) c_{2, l}\right)+\frac{\partial \rho_{i, l}}{\partial t}+\vec{v} \cdot \operatorname{gradc}_{2, l}+f_{i, l}\left(x, y, z, t, c_{2, l}, c_{1, l}, \rho_{i, l}\right)= \\
& =\sum_{k=1}^{n}\left(\operatorname{div}\left(D_{k l}\left(x, y, z, t, c_{2, l}(x, y, z, t), c_{2, l}(x, y, z, t-\tau), b\right)\left(\operatorname{gradc}_{2, l}\right)\right)\right), \\
& \cdots \\
& \frac{\partial}{\partial t}\left(\sigma(x, y, z, t, \rho) c_{n, l}\right)+\frac{\partial \rho_{i, l}}{\partial t}+\vec{v} \cdot \operatorname{gradc}_{n, l}+f_{i, l}\left(x, y, z, t, c_{n, l}, \ldots, c_{1, l}, \rho_{i, l}\right)= \\
& =\sum_{k=1}^{n}\left(\operatorname{div}\left(D_{k l}\left(x, y, z, t, c_{n, l}(x, y, z, t), c_{n, l}(x, y, z, t-\tau), b\right)\left(\operatorname{gradc}_{n, l}\right)\right)\right), \\
& \frac{\partial \rho_{i, l}}{\partial t}=\beta_{i, l}\left(x, y, z, t, c_{i, l}\right) \sum_{i=1}^{m} k_{i} c_{i, l}-\alpha_{i, l}\left(x, y, z, t, \rho_{i, l}\right) \rho_{i, l}+f_{i, l}^{*}\left(x, y, z, t, c_{i, l}, \rho_{i, l}\right)+ \\
& +\sum_{k=1}^{n}\left(\operatorname{div}\left(D_{k l}^{*}\left(x, y, z, t, \rho_{i, l}(x, y, z, t), \rho_{i, l}(x, y, z, t-\tau), b^{*}\right)\left(\operatorname{grad}_{i, l}\right)\right)\right), i=\overline{1, n} ; l=\overline{1, m}, \\
& \quad \vec{v}=\kappa(x, y, z, t, \rho) \operatorname{grad} \varphi, \operatorname{div} \vec{v}=0,
\end{aligned}
$$


where $c=\left(\begin{array}{l}c_{1,1}, \ldots, c_{1, m} \\ \ldots \\ c_{\mathrm{n}, 1}, \ldots, c_{n, m}\end{array}\right)-$ concentration of impurities in the filtered liquid, $\rho=\left(\begin{array}{l}\rho_{1,1}, \ldots, \rho_{1, m} \\ \ldots \\ \rho_{n, 1}, \ldots, \rho_{n, m}\end{array}\right)$ - concentration of sediment in loading, $\beta=\left(\begin{array}{l}\beta_{1,1}, \ldots, \beta_{1, m} \\ \ldots \\ \beta_{\mathrm{n}, 1}, \ldots, \beta_{n, m}\end{array}\right)-$ coefficient of impurity particles adsorbed per unit time, $\alpha=\left(\begin{array}{l}\alpha_{1,1}, \ldots, \alpha_{1, m} \\ \ldots \\ \alpha_{\mathrm{n}, 1}, \ldots, \alpha_{n, m}\end{array}\right)-$ coefficient of desorbed particles precipitate per unit time, $D_{k l}=\left(D_{k l i j}\right)_{n \times m}, D_{k l}^{*}=\left(D_{k l i j}^{*}\right)_{n \times m},-$ diffusion in fluid and sediment, $\kappa=\left(\kappa_{i j}\right)_{n \times m}$ - coefficient of filtering, $\vec{v}-$ vector of speed filtering, $f_{k l}=\left(f_{k l i j}\right)_{n \times m}, f_{k l}^{*}=\left(f_{k l i j}^{*}\right)_{n \times m}$ - functions of mutual influences, $\sigma$ - the porosity of the medium.

\section{CONCLUSION}

That's way analysis of problems of mathematical modeling of filtering processes through porous medium, review of existing models and methods of mathematical modeling processes of purification of liquids from multicomponent contamination by filtrating in a porous medium, it has been stated that scientific and practical problems are relevant mathematical models of processes of purification of liquids from multicomponent contamination. by filtration, which take into account the inverse effect of process characteristics on environmental characteristics. Also diffusion-mass transfer perturbations and development of numerically asymptotic methods for solving the corresponding nonlinear regular and singularly perturbed boundary-value problems, as well as, on the basis of solving model problems, construction of automation systems for the respective treatment systems and complexes are relevant.

\section{REFERENCES}

[1] Aiba S. A masstransfer in porous media / Aiba S., Humphrey A. E., Milles N. Z. // Biochem. Eng. - 1965. - V. 9. - P. 1892-1904.

[2] Andrii Safonyk Mathematical modelling process of liquid filtration taking into account reverse influence of process characteristics on medium characteristics / Andrii Safonyk, Andrii Bomba // International Journal of Applied Mathematical Research. - Vol. 4. - No. 1. - 2015. P. 1-7.

[3] Baumann E. R. Polyelctrolyte coatings for filter media / Baumann E. R., Oulman Ch. S. // Ind. Water Eng. - 1971. - V., № 5. - P. 22-25.

[4] Bomba A. Mathematical modeling of aerobic wastewater treatment in porous medium / Bomba Andrij, Safonyk Andrij // Zeszyty Naukowe WSInf Vol 12, Nr 1. - 2013. - P. 21-29.

[5] Safonyk A. P. Computer simulation of cleaning fluid from multicomponent pollution by multilayer filter / Safonyk A. P. // Proceedings of the International Scientific Conference "Modern Problems of Mathematical Modeling and Computational Methods". Rivne : EPD RSHU, 2015. - P. 228.

[6] Safonyk A. Simulation of fluid filter n-layer filters multicomponent with the pollution in view / Andrij Safonyk, Oleksandr Naumchuk // Informatyka Automatyka Pomiary w Gospodarce i Ochronie Srodowiska 4/2014.-Poland, P. - 28-31 